\title{
FRANZÖSISCHE REISENDE DES 19. JAHRHUNDERIS IN FRANKFURT
}

Die Geschichte der Reisen ist die Geschichte der Reiserouten und der Reiseziele. So selbstverstăndlich dies klingen mag, es erklărt doch, warum bestimmte Orte und Gegenden erst von einer bestimmten Zeit an die Aufmerksamkeit der Reisenden auf sich zogen. Die klassische Reise und noch die berühmte Kavalierstour des 18 . Jahrhunderts dient ja nicht dem touristischen Verweilen, nicht dem Zick-Zack in fremden Landen, wie sich spăter die Romantiker Théophile Gautier oder Rodolphe Toepffer ausdrücken werden, sondern dem lăngeren Aufenthalt in den großen gesellschaftlichen Zentren, die zugleich als kunstlerische und literarische Mittelpunkte gelten und in denen der Fremde mit dem kulturellen Leben der besseren Gesellschaft vertraut zu werden versucht. Hin- und Ruckweg, Zwischenaufenthalte, wofern sie nicht selbst von hochrangiger Bedeutung sind, sind weitgehend festgelegt und spielen meist keine besondere Rolle ${ }^{1}$. Gesellschaftliches Zentrum aber heißt bis zum Ausgang des 18. Jahrhunderts höfisches Zentrum. Man geht nach Paris und London, Madrid und Rom, Berlin und Wien, Konstantinopel und Sankt Petersburg, wenn man sich mit den Verhaltnissen des jeweiligen Landes vertraut machen will. Und hier - wie noch Madame de Staél in Weimar oder Wien - sucht man das Charakteristische und Repräsentative, das als zusammenfassender Ausdruck der Kultur, gleichsam als Kulturbarometer, dient. Mit einem Beispiel : noch Chateaubriand, als Diplomat 1821 an dem Berliner Hof unterwegs, hat für « la route trop battue de Francfort $"{ }^{2}$ kein Auge; in seinen Mémoires d'outre-tombe schreibt er lakonisch : " Francfort, cité des Juifs ne m'arrêta que pour une de leurs

1. Dazu und zum Folgenden, cf. Friedrich Wolfzetrel, Ce Désir de voyage cosmopolite. Wege und Entwicklung des französischen Reiseberichts im 19. Jh., Tubingen, Niemeyer, 1986, p. 18 sq. 632 .

Revue de synthèse : IV S. No 1-2, janv.-juin 1992. 
affaires : un change de monnaie ${ }^{3}$. Erst der romantische Reisende sucht das jeweils Besondere, achtet auf das Unterschiedliche und setzt das Gesamtbild aus vielen Einzelheiten zusammen. Erst so werden einzelne Städte beschreibenswert, werden geschlossene Landschaften Objekte von Reiseberichten. Z. T. unabhängig von den großen Zentren bilden sich historisch, geographisch, ja symbolisch deutbare Konfigurationen heraus : das maurische Spanien und nicht nur Madrid, die norditalienischen Seen und nicht mehr nur Mailand, die Toskana und nicht mehr bloß Florenz, die Pyrenäen, der Lake Districkt... man möchte endlos fortfahren. Für uns aber ist wichtig : auch das Rheinland.

Es scheint nämlich, daß die wachsende Bedeutung einer Stadt wie Frankfurt für französische Reisende seit den 30er Jahren des 19. Jahrhunderts mit der - gegenüber den Engländern und Italienern - verspäteten Entdeckung des romantischen Rheins zu tun hat ${ }^{4}$. Die Rheinreise stellt lange nach dem epochalen Buch Madame de Staëls, De l'Allemagne (1813) eine Art Sprungbrett für die französische Deutschlandreise überhaupt dar, die erst jetzt einen anerkannten kulturellen und literarischen Status erhält und eine eigene Tradition bildet. 1831 bringt der Hofrat Aloys Schreiber in Heidelberg ein Manuel des voyageurs sur les bords du Rhin heraus, das zur Standardlektüre französischer Reisender wird; nur ein Jahr später erscheint die französische Fassung der Rheinreise von Mainz bis Köln von Professor J. A. Klein unter dem Namen des neuen Verlegers Karl Bädecker, und es dauert nicht lange, bis in der renommierten Reihe der Guides Joanne die Bords du Rhin einen objektiven Reiseführer moderner Prägung bereitstellen. Zwischen Süd- und Norddeutschland, parallel zu Mainz und fast genau in der Mitte des Buches steht hier die Beschreibung der Stadt Frankfurt und ihrer Umgebung, "chef-lieu de la république de ce nom " - der Status als freie Reichsstadt war ja 1815 wiederhergestellt worden - , mit nicht ganz 84000 Einwohnern, davon 11000 Katholiken und 4000 Juden, wie der Führer angibt ${ }^{5}$. Damit wird Frankfurt zur Drehscheibe jeder an der romantischen Rheinroute orientierten Reise, wie bei Nerval, Alexandre Dumas, Victor Hugo, Jules Janin, Désiré Nisard und später den Reisenden des Second Empire und der Dritten Republik.

3. Ibid., p. 36.

4. Cf. mein Nachwort zu Victor Hugo, Rheinreise, Frankfurt, Societätsverlag, 1982, ferner die Dissertation von Theodor COHNEN, Der Rhein in der französischen Literatur, Bonn, 1926 ; Walter OTtEnDORF-SimRock, Die Stimme des Rheins. Der Strom im Spiegel der Dichter des 18. und 19. Jahrhunderts, Honnef, Peters, 1956 ; Jorg-Ulrich FECHNER, Erfahrene und erfundene Landschaft. Aurelio dé Giorgi Bertolas Deutschlandbild und die Begrïndung der Rheinromantik, Opladen, Westdeutscher Verlag, 1954.

5. Trains de plaisir aux bords du Rhin, Guides Joanne, Paris, Hachette, 1863, p. 217. 
Die Reiserouten und geographischen Verhältnisse in einem bewußt ästhetisch arrangierten Reisebericht sind über ihren Faktizitätscharakter hinaus fast immer interpretierbar. Die genannte Drehscheibenfunktion gilt daher durchaus auch in einem ästhetischen Sinne. Ergănzt werden kann hier aber gleich ein komplementärer Aspekt, den ich mit der Parallele Mainz-Köln bereits angedeutet habe. Neben der vertikalen spielt nämlich auch die horizontale Achse der beiden Städte links und rechts des Rheins fast von Anfang an eine spezifische Rolle in bezug auf die quasi-mythische Rheingrenze, die schon bei Madame de Staël als "solennelle " ${ }^{6}$ bezeichnet wird und die später Victor Hugo die Grundlage für seine große Frankreich-Deutschland-, Linksrhein-Rechtsrheinsymbolik liefert. Mainz nämlich, die einstige Hauptstadt des Départements Mont-Tonnerre und Brückenkopf des Napoleonischen Reiches scheint für den französischen Reisenden häufig bereits die Heimat vorwegzunehmen - so bei Hugo, Michelet und noch am Anfang des 20. Jahrhunderts bei Jules Huret -, freilich nicht immer nur im positiven Sinn, wenn etwa der liberalkonservative Sozialreformer Nisard 1836 suffisant notiert : « mais il faut dire que pour la propreté, Mayence n'est pas une ville allemande, mais bien plutôt française $"{ }^{7}$. In freundlicher und ausgleichender Perspektive dient die Parallele Mainz-Frankfurt einem Xavier Marmier 1860 dazu, den Unterschied zwischen der Mainzer Revolutionsbegeisterung und der traditionsbewußten Haltung Frankfurts zu betonen. Aber weil Frankfurt so "deutsch " ist, kann es als charakteristisch verstanden werden. Es repräsentiert merkwürdigerweise in zunehmendem Maße - neben wichtigen Stădten wie Leipzig, München und besonders Berlin - eine Dialektik von Tradition und Innovation, Alt und Neu, Pittoreske und Luxus, die wie ein Leitmotiv die verschiedenartigen Reiseerinnerungen durchzieht. Frankfurt, symbolischer Abschluß der romantischen Rheinroute oder aber Endpunkt der lieblichen sũddeutschen Landschaft und selbst, wie der Guide Joanne vermerkt, " une des plus belles villes d'Allemagne " ${ }^{8}$, wird dadurch nămlich mit der modernen Stadt par excellence, Berlin, vergleichbar und reflektiert zugleich eine Faszination oder Problematik, die den französischen Reisenden offensichtlich unweigerlich an Paris, wenn nicht an London erinnert. Über die genannten symbolischen Koordinaten hinaus erhält die Stadt mithin einen modellhaften Status. Zitieren wir noch einmal Nisard : "Francfort étonnerait bien ceux qui croient que toute la civilisation est à Paris et à Londres ${ }^{9}$. Es klingt wie ein Echo, wenn der elsäs-

6. De l'Allemagne, I. XIII.

7. Anonyme (= Désiré Nisard), Promenades d'un artiste. Bords du Rhin, Hollande, Belgique, Paris, Jules Renouard, 1935, p. 121.

8. Op. cit. supra n. 5, p. 217.

9. D. NisARD, op. cit. supra n. 7, p. 107. 
sische Journalist Jean-Jacques Weiss 1886 die Stadt als « une ville d'élégance, liée à Paris par tant d'affinités " ${ }^{10}$ kennzeichnet, oder wenn noch Jules Huret in seiner großartigen Deutschlandenquête von 1907 der Stadt bewundernd ihren " air de capitale " " bescheinigt. In einem Zeitalter, das zwischen romantischer Nostalgie und Fortschritts-, Komfort- und Luxusglăubigkeit hin- und herschwankt und zunehmend den heutigen Entwicklungsmaßstab zum Wertkriterium erhebt, erhält die alte Reichsstadt, Messe- und Bankenstadt, die untrennbar mit dem Namen Rothschild verbunden ist, sowohl zivilisationsgeschichtlich wie national gesehen repräsentativen Rang. Sie ist nicht nur wie Aachen, Köln, Heidelberg, Stuttgart, Leipzig, Dresden, usw., Ausdruck eines besonderen Geistes und einer besonderen, durch die Geschichte geprăgten Physiognomie; sie steht daruber hinaus auch für ein Deutschlandbild, ja sie wird selbst, als ehemalige Kaiserstadt und jetzige Bankenstadt, nachdem die höfischen Zentren an Bedeutung verloren haben, zum Gradmesser der deutschen Entwicklung, eine Rolle, die selbst nach der Reichsgründung und der stürmischen Entwicklung Berlins in französischen Augen erhalten bleibt.

Obwohl die positiven Bewertungen bei weitem überwiegen, sind die Gewichte naturgemăß verschieden verteilt, ja ăndert sich die Blickrichtung, mit welcher die angesprochene Dialektik zwischen Alt und Neu konstatiert wird. Die Stadt hat ja den Verfechtern beider Tendenzen etwas zu bieten : " elle se recommande tout à la fois à ceux qui aiment les villes neuves et à ceux qui se passionnent surtout pour les vieilles villes ${ }^{12}$, schreibt der genannte Reisefuhrer. Die Haltung der großen Romantiker ist in diesem Punkt zumindest ambivalent, und eine gewisse Desillusion ist hăufig nicht zu verkennen. Ein Gérard de Nerval, der wie kein anderer zur Vermittlung deutschen Geistes in Frankreich beigetragen hat und ein enthusiastisches Bild des alten Germanien, "notre mère à tous ", bewahrt, zeichnet in seinem Lorely überschriebenen Reisebericht aus den 40er und 50er Jahren ein sehr positives Bild, in dem musikalische (die Aufführung der Faust-Oper von Spohr) und literarische Erinnerungen (Goethe, Wilhelm Meister) zu einer großen « féerie » gesteigert werden : Von der Mainlust aus, schreibt der Autor

« on admire une des plus belles perspectives du monde, la vue de Francfort s'étendant sur la rive gauche, avec ses quais bordés d'une forêt de mâts, et du

10. Jean-Jacques Auguste Weiss, Aux pays du Rhin. Metz, Homboung les-Bains, autour de Hombourg, Francfort, Strasbourg et l'Alsace, Paris, Charpentier, 1886, p. 109.

11. Jules Huret, En Allemagne, Rhin et Westphalie, Paris, Fasquelle, 1907, p. 50.

12. Op. cit. supra n. 5 , p. 217. 
faubourg de Sachsenhausen situé à droite, qu'un pont immense joint à la ville; des palais aux riantes terrasses, de longues suites de jardins et des restes de vieilles tours embellissent les bords du fleuve, où le soleil couchant se plonge comme dans la mer, tandis que la chaine du Taunus ferme au loin l'horizon de ses dentelures bleuâtres " ${ }^{13}$.

Und er schließt mit einem visionären Gesamtbild, das im Stil Victor Hugos Geschichte, Mensch und Natur vereinigt :

" C'est une des plus belles et complètes impressions dont le souvenir est éternel ; une vieille ville, une magnifique contrée, une vaste étendue d'eau : spectacle qui réunit dans une harmonie merveilleuse toutes les auvres de Dieu, de l'homme et de la nature ${ }^{14}$.

Eine leise Melancholie mischt sich freilich kurze Zeit spăter in dieses begeisterte Bild einer im wesentlichen noch intakten, gotisch geprägten Stadt : Als Nerval die Stadt wieder besucht und vor allem das Goethedenkmal von Schwanthaler bewundert und das Goethe-Haus besucht, notiert er - mit einem Seitenblick auf Victor Hugo, der inzwischen wie wir sehen werden - eine weniger begeisterte Beschreibung gegeben hat : " quelque chose d'attristant plane aujourd'hui sur la cité libre, qui fut si longtemps le coeur du vieil empire germanique $"{ }^{15}$. Frankfurt erscheint nur noch in der Perspektive des Abschieds. In der geheimnisvollen Reiseerzählung Angélique (1854), in der die Suche nach einem alten Buch als labyrinthische Identitătssuche vorgeführt wird, hat dann der Ton offensichtlich ganz umgeschlagen. Frankfurt ist noch immer die Bücherstadt, und doch erscheinen das plotzliche Auftauchen und der Erwerb eines alten franzősischen Buches, der Geschichte des Abbé de Bucquoy, inmitten des "luxe inoui " der Stănde und Schaufenster als merkwürdig inkongruent, ja fast wie eine strafbare Handlung, die das Ich zum Flüchtling und Ausgestoßenen aus einer merkantilen, polizeilich überwachten Welt macht ${ }^{16}$. Das Wahnsystem des Autors spiegelt eine Erfahrung, die der Historiker Michelet sehr viel nüchterner, doch im Kern ahnlich formuliert :

« Me voici dans Francfort, dans cette ville que je n'ai pas revue depuis 1828. Que de choses changées en moins de quinze ans ! [...] Francfort où j'ai remué tant de livres, d'idées, me semble être uniquement aujourd'hui, le grand

13. Lorely. Souvenirs d'Allemagne, Euvres de Gérard de Nerval, ed. par Albert Btouin et Jean Richer, Paris, Gallimard, « Biblioth. de la Pléiade », 1960-1961, t. IL, p. 765.

14. Ibid.

15. Ibid., p. 758.

16. Ibid., I, p. 160. 
marchẻ d'argent pour toute l'Europe. Les vieux souvenirs historiques s'effacent $"{ }^{17}$.

Das Fremdheitserlebnis steht hier am Ende der romantischen Initiation - schon am nächsten Tag reist der Autor nach Mainz, einem Stück Frankreich - « un peu de la Patrie » - weiter. Frankfurt wird so zum symbolischen Endpunkt und bezeichnet den entschwindenden Horizont des romantischen Deutschland. Alexandre Dumas, der romantischen ebenso wie der humoresken Tradition des Reisegenres verpflichtet, kleidet die nămliche Erfahrung in eine witzige Anekdote : Auf der Suche nach einem französischsprechenden Frankfurter wendet er sich an einen urbanen Bankier im Ruhestand, um ihn nach der « maison de Goethe » zu fragen - dem Autor selbst widmet er einige Zeilen später einen begeisterten Nachruf; " la maison Goethe, la maison Goethe », antwortet der Bankier verlegen, "mit diesem Haus habe ich während meiner Berufszeit nie zu tun gehabt $"{ }^{18}$. Die zentrale Stelle in der Stadtbeschreibung erhält dann folgerichtig nicht das Goethehaus, sondern der fabulöse Aufstieg der Rothschilds aus dem ärmlich-biederen Milieu der Judengasse.

Die merkwürdige Haltung Victor Hugos in seinem Frankfurt-Kapitel von Le Rhin (1840) ${ }^{19}$ erklärt sich vielleicht durch diese heimliche Desillusion, sucht doch der Dichter ohnedies nicht das aktuelle, politische und soziale Bild des Nachbarlandes, sondern den traumhaften Abglanz einer Essenz, die in Geographie und Geschichte, Märchen und Sagen wiederzufinden ist. Es ist, als verzichte Hugo unbewuBt auf jede Gesamtwertung, als schließe er die Augen vor dem angesprochenen Kontrast, um sich ganz der intimen Begegnung mit einer verwaisten Vergangenheit hinzugeben. Lediglich die bizarren Karyatiden der neureichen Stadthăuser und das pantagruelische Gemälde der üppigen Metzgerläden evozieren wie nebenbei eine negative Phantasmagorie von Luxus, Ubberfluß und Roheit. Doch zentral ist die einsame Besichtigung des Kaisersaals, dessen 45 Lücken für Kaiserbüsten genau mit dem letzten Kaiserbildnis bei der Auflösung des Heiligen Römischen Reiches ausgefüllt gewesen seien und "l'histoire même de l'Allemagne " ${ }^{20}$ symbolisierten. Den Irrtum rückt freilich dann Nerval zurecht, indem er diskret die Hugoschen Büsten als Illusionsmalerei entlarvt. Aber Victor Hugo braucht die Aura der glanzvollen Vergangenheit, er taucht seine Besichtigung des Römer in eine

17. Jules Michelet, « En Allemagne », 1842, Revue des Deux Mondes, 121, 1894, p. 584.

18. A. Dumas, Aux bords du Rhin, Alexandre Dumas illustré, Paris, s.d., chap. xxII, p. 68 sq., hier p. 69.

19. V. Hugo, Le Rhin, Strasbourg, Bueb et Reumaux, 1980, chap. xxIv, p. 275 sq.

20. Ibid., p. 280. 
Atmosphäre der Stille und der Verlassenheit; der anfangs verschlossene Kaisersaal deutet auf eine verschlossene Vergangenheit, zu der nur noch die dichterische Intuition Zugang findet. Und so klingt die Episode in eben solcher Stille mit dem Rundblick vom Pfarrtum aus, der gerade nicht den Überblick über die moderne Realităt, sondern eine tiefere, fast visionäre Einheit von Stadt und Landschaft in dem Spiel von Licht und Wolken meint : "Cette ville et cet horizon étaient admirables ainsi " ${ }^{21}$. Die Vision mündet wohl in die Gegenwart, aber eine Gegenwart des jederzeitlichen kleinen Glücks und stillen Lebens, das nur als Kontrast und Protest gegen den eingangs angedeuteten Fortschritt einer materiellen Zivilisation gedeutet werden kann. Beim Umschreiten der Kirchturmplattform entdeckt der Dichter eine Art Dachgartenidylle, eine einfache, ältere Frau und ein hübsches vierzehnjähriges Mädchen, vereint in einem " petit monde doux et heureux " ${ }^{22}$. Die stolze Geschichte der einstigen Reichsstadt wird korreliert mit dem kleinen Glück des Menschlichen : "Cette fière ville impériale, qui a soutenu tant de guerres [...] est aujourd'hui dominée et couronnée par l'humble foyer d'une humble femme, d'où sort un peu de fumée ${ }^{23}$.

Kehren wir dieses Bild um, so ergibt sich das weithin positive Bild, das der Musiker Hector Berlioz auf seiner ersten Deutschlandtournee 1843 von Frankfurt zeichnet :

« Voilà une ville charmante et bien éveillée! Un air d'activité et de richesse y règne partout; elle est en outre bien bâtie, brillante et blanche comme une pièce de cent sous toute neuve, et des boulevards plantés d'arbustes et de fleurs dans le style des jardins anglais, forment sa ceinture verdoyante et parfumée ${ }^{24}$.

Hatte nicht schon Alexandre Dumas staunend vermerkt, daß die Stadt die alten Ringwälle und Mauern durch Grulnanlagen ersetzt hatte :

« si bien qu'avec ses maisons peintes en blanc, en pistache et en rose, Francfort ressemble à un énorme bouquet de camélias tout entouré de bruyères " - « charmant labyrinthe que les bourgeois et leurs familles peuplent tous les jours à cinq heures $"{ }^{25}$.

Eine Beschreibung, die man - trotz des heute noch vorhandenen Grüns - sicher nur mit einer gewissen Melancholie zur Kenntnis neh-

21. Ibid., p. 283

22. Ibid.

23. Ibid., p. 284.

24. Hector Berloz, Mémoires, éd. par Pierre Criron, Paris, Gamier-Flammarion, 1969, « Premier voyage en Allemagne ", p. 56.

25. Aux bords du Rhin, op. cit. supra n. 18, p. 68. 
men kann. Spätere Besucher werden kaum anders urteilen und z. B. wie Victor Tissot 1877 den Palmengarten bewundern, nicht ohne im übrigen dessen antidemokratische, für die damalige Zeit enormen Eintrittspreise (10 Mark) hervorzuheben. Doch bleiben wir noch bei dem positiven Register. Der Philosoph und Romancier Hippolyte Taine, der Deutschland kurz vor dem Krieg von 1870/1871 besucht und als einer der ersten ein durchweg negatives Deutschlandbild entwirft, konstatiert dennoch :

« Belle ville bien propre et soignée, ce Francfort. Elle a été cité libre, presque république, jusqu'en 1866 ; et on prend soin de sa patrie. Mais jusque dans ses larges rues, dans le Zeil, il y a des traces du Moyen Âge... ${ }^{26}$.

Vielleicht sollte die Stadt sich etwas darauf zugute halten, daß sie gerade in den wenig deutschfreundlichen Deutschlandreisen französischer Autoren nach $1871^{27}$ relativ oder sogar besonders gut wegkommt. Ein Ernest Feydeau etwa, der Vater des bekannten Komødienautors und Rivale Flauberts, zeichnet ein fast karikaturales Bild des neuen Deutschen Reiches und seiner durchweg primitiven und barbarischen Bewohner, nimmt aber dennoch unter die " charmantesten Städte » neben Köln, Baden-Baden und Bad Homburg auch Frankfurt auf. Ja, den Frankfurtern wird die Ehre zuteil, zuweilen die Ausnahme von der Regel zu repräsentieren, wonach $"$ il serait impossible de rencontrer sur la terre une race qui fût à la fois aussi brutale et aussi grossière que la race allemande $" 28$. Ein platonischer Flirt mit der reizenden Augusta und ein kurzes Gespräch mit einer vornehmen Frankfurter Dame bei der Rückkehr an der Gare du Nord in Paris bestätigen dies. Auch ein Victor Tissot, der Ende der 70er Jahre eine große Erkundungsreise durch das - mithilfe französischer Kriegsentschädigungen - reich gewordene « pays des milliards " unternimmt, sieht in Frankfurt die große Handels- und Messestadt, deren « côté mercantile ne gâtait pas le côté pittoresque " ${ }^{29}$. Der bereits erwăhnte Journalist Jean-Jacques Weiss schließt seinen Bericht ein Jahrzehnt spăter mit dem Ausruf : «Que voilà une ville vivante et opulente, qui sait mettre la vie en œuvre et en jouir! ${ }^{30}$. Und Jules Huret urteilt 1907 : «Voici l'une des villes les plus intéressantes d'Allemagne, l'une des plus agréables à habiter, quoi qu'en puissent penser tels Français que j'ai trouvés là, les ailes nouées ${ }^{31}$.

26. Hippolyte TAINe, Voyage en Allemagne, 1870, RdM, 60, 1920, p. 449-489, hier p. 451.

27. Cf. F. Wolfzermel, Das entzauberte Deutschland. Französische Reiseberichte zwischen 1870 und 1914, in Grenzgdnge. Kulturelle Begegnungen zwischen Deutschland und Frankreich, éd. par Hans T. SIEPE, Essen, Die blaue Eule, 1988, p. 64-82.

28. Emest Feydeau, L'Allemagne en 1871, impressions de voyage, Paris, Lévy, 1872, p. 64.

29. Victor Tissor, Voyage au pays des milliards, Paris, E. Dentu, 1872, p. 65.

30. Aux pays du Rhin, op. cit. supra n. 10, p. 119.

31. J. HuRET, op. cit. supra n. 11, p. 44. 
Ein besonderer Aspekt, der natürlich bei Berlioz im Vordergrund steht, verdient noch betont zu werden : die Musikstadt Frankfurt. Die Erlebnisse, die Berlioz beschreibt, sind nicht nur positiv. Dennoch gefallt es ihm. $\mathrm{Zu}$ spät benachrichtigt, erfährt er bei seiner Ankunft in der Stadt vom Musikdirektor Guhr, daß ein gerade gut gehendes populäres Programm keinen Raum für seine geplante Konzertaufführung läßt. Er hat jedoch Gelegenheit, einer Aufführung des Fidelio beizuwohnen, und zeigt sich ebenso begeistert über die Qualität wie konsterniert über den geringen Beifall des "public de bourgeois " ${ }^{32}$. Nebenbei erwähnt er lobend die Gesangsakademie von Sankta-Cäcilia. Was hier jedoch nur angedeutet ist, wird vierzig Jahre spåter von Weiss ausfuhrlich entwickelt. Frankfurt und Bad Homburg stehen in Au Pays du Rhin für Theater und Musik. Wie allen Autoren nach 1871 geht es Weiss um einen kulturellen Vergleich zwischen Frankreich und Deutschland, so da $B$ Frankfurt wie von selbst die eingangs genannte Rolle eines repräsentativen Kulturbarometers zukommt. In diesem Rahmen nun verhehit der Autor nicht seine Bewunderung sowohl für den künstlerischen Standard als auch für die Qualität und Kompetenz des Publikums, die sich offenbar seit Berlioz merklich gebessert hat : "en matière théâtrale, les suffrages du public allemand ne sont pas du tout à dédaigner ${ }^{33}$. Er erlebt die große Komödienschauspielerin Clara Delio und eine Aufführung des Freischütz, die er sogar über die Pariser Oper zu stellen wagt. Was aber besonders ins Auge fällt, ist sein Lob für den Frankfurter Bürgersinn - vor allem in bezug auf die Subventionierung der Neuen Oper, die 1880 fertiggestellt wurde - und für die fortschrittliche Bühnentechnik und Bauhygiene, in denen Frankfurt Paris überlegen sei. Die Bühnenmaschinerie etwa, die er fachmännisch besichtigt, erscheint ihm " tout uniment " als « la huitième merveille du monde $"{ }^{34}$; die Sicherheitsbestimmungen und -vorkehrungen sind bewundernswert, und das ganze Gebäude ist mit seinem ausgeklügelten Belüftungs- und Heizungssystem «comme un corps où le sang circule ": "Quel plaisir d'être assis dans sa stalle à l'Opéra de Francfort! comme on s'y sent en sûreté et en hygiène! $"{ }^{35}$.

Wir sind damit erneut bei dem Thema Fortschritt und Entwicklung, das wir noch einmal kurz chronologisch anhand der wichtigsten Zeugnisse des letzten Drittels des 19. Jahrhunderts verfolgen wollen. Bei diesem - wie wir sahen, bereits romantischen Thema geht es ja auch um die Identität der einstigen Kaiserstadt und Reichsstadt. Chronologisch und motivisch in der Mitte steht der schon flüchtig erwähnte Voyage pit-

32. H. BerLIOZ, op. cit. supra n. 24, II, p. 61.

33. Aux pays du Rhin, op. cit. supra n. 10, p. 86.

34. Ibid., p. 99.

35. Ibid., p. 118. 
toresque en Allemagne septentrionale (1860) des Weltreisenden Xavier Marmier; er sieht die Stadt mit damals 70000 Einwohnern fast programmatisch unter dem Gesichtspunkt des fruchtbaren Kontrasts zwischen dem altdeutschen Geist und fröhlich kosmopolitischer Öffnung, der Rolle als Bundeshauptstadt und den Handelsbeziehungen " avec le monde entier " ${ }^{36}$, der noch vorhandenen Judengasse, "qui fait un étrange contraste avec les quartiers qui l'avoisinent ${ }^{37}$, und der mondänen Zeil, einer internationalen Flanierstraße, die ihre Rolle auch den günstigen Eisenbahnverbindungen und den guten Hotels verdanke kurz der geglückten Verbindung von "son élégance aristocratique " und "son activité industrielle", des "mouvement intelligent" mit dem " bien-être matériel ${ }^{38}$. Romantisches Deutschlandbild und zeitgenössischer Fortschrittsoptimismus werden hier nicht als Gegensatz, sondern als Ergänzung begriffen, obwohl oder gerade weil der Betrachter die Stadt auch früher immer wieder besucht hat : denn er liebt sie, wie er sagt, wegen ihrer Vielfalt : « Je l'aime, cette impériale cité, pour la variété de ses points de vue... $"{ }^{39}$.

Andere Stimmen klingen ambivalenter. Am unmißverständlichsten zeigt dies wohl Victor Tissot, der noch das romantische Deutschland und seine idealistische Tradition kennengelernt hat und daher umso heftiger auf die traumatische Erfahrung von 1870/1871 reagiert. Das GoetheHaus und seine Botschaft sind für ihn der Inbegriff einer vergangenen bürgerlichen "honnêteté " und Innerlichkeit, die der neuen Schau- und Protzsucht des Wilhelminischen Reiches Platz gemacht habe : "Que les Allemands sont donc pratiques, - et quelle poésie matérialiste que la leur ! ${ }^{40}$. Man müsse sich daher in jeder Hinsicht beeilen, um noch etwas von dem alten Frankfurt zu erhaschen :

«Il faut se hâter de voir Francfort; ce n'est pas une ville qui se meurt, c'est au contraire une ville qui ressuscite; mais aux yeux de l'archéologue, de l'historien et de l'artiste, cette résurrection équivaut à la mort. Encore dix ans de transformations successives, et l'antique cité impériale n'aura conservé de son passé que des photographies ${ }^{41}$.

Die Judengasse existiert nicht mehr; der restaurierte Kaisersaal wirkt merkwürdig künstlich, und der Römer, « cette place, qui renferme toute

36. Xavier Marmier, Voyage pittoresque en Allemagne septentrionale, Paris, Morizot, 1860 , p. 458.

37. Ibid., p. 459.

38. Ibid., p. 458.

39. Ibid.

40. V. Tissor, op. cit. supra n. 29, p. 68.

41. Ibid., p. 60. 
l'histoire d'Allemagne, n'a pu se soustraire au souffle de transformation qui passe sur Francfort et en efface les derniers traits de poésie et d'originalité " ${ }^{42}$. Umgekehrt : " La vie moderne s'y étale dans toutes ses élégances, et même dans ses extravagances $"{ }^{43}$. Dies ist sicherlich nicht nur der enttäuschten Haltung des Romantikers zuzuschreiben. Was Tissot vor allem sieht, ist der langsame Identitätsverlust der alten Reichs- und Kaiserstadt, die sich in den preußischen Machtstaat eingliedert. Noch freilich vermerkt der Autor die antibismarckische Presse, die Erinnerungen an die preußische Besatzung und Ausblutung 1866, als « Francfort ne fut pas mieux traitée qu'une ville française " unter dem Regiment der Generäle Vogel von Falkenstein und Manteuffel ${ }^{44}$, noch spricht er von der "illusion d'une ville française, occupée par une garnison prussienne " und bezeichnet die Stadt emphatisch als "la ville irréconciliable "

Von solchen politischen Tönen ist in der Folge nicht mehr viel zu hören. Weiss kann sich noch daran erinnern, 1869 die Losung «PreuBen ! Nieder ! Nieder ! " gehört zu haben. Aber :

«Que Francfort est changé de ce que je l'ai vu en 1869 ! [...] Francfort est à présent fondu sans réserve et sans arrière-pensée avec la Prusse et avec l'empire. Si Francfort regrette quelque chose, ce n'est pas le Bund. Francfort regrette de n'être pas demeuré le premier marché financier de l'Allemagne ; Berlin lui a ravi ce rang $"{ }^{46}$.

Dennoch hat sich der Abschied von politischer Nostalgie bezahlt gemacht : " Francfort est une maison de banque qui occupe un emplacement unique sur le continent, à égale distance des bourses de Paris, de Berlin, de Vienne, de Hambourg et d'Amsterdam $"{ }^{47}$. Auch Weiss vermerkt das Einreißen der Judengasse : « Je suis arrivé juste à temps pour revoir une dernière fois la maison croulante, berceau de Rothschild... $"{ }^{48}$. Er geht auf die Stadterneuerung seit 1870 ein, die Ausweitung nach allen Richtungen, die Verlängerung der Zeil, die Anlage der Kaiserstraße und den Bauboom des Westend mit seiner «diversité grasse sans discordance ${ }^{49}$ und den protzigen Fassaden, « la décoration pour la décoration, l'art pour l'art ${ }^{50}$; und endlich vergleicht er die üppigen Auslagen der Kaiserstraße mit denen von Paris und sieht bereits die unangefoch-

42. Ibid., p. 61 sq.

43. Ibid., p. 71.

44. Ibid., p. 74.

45. Ibid., p. 71.

46. J.-J. WeIss, Aux bords du Rhin, op. cit. supra n. 18, p. 101.

47. Ibid., p. 102.

48. Ibid.

49. Ibid., p. 104.

50. Ibid., p. 106. 
tene Geltung der Pariser Mode und Luxusindustrie in Gefahr : " Nous régnons encore par la mode ; nous ne régnons plus seuls, ni d'un sceptre incontesté $"{ }^{51}$. Entgegen einem in Frankreich weit verbreiteten Irrtum repräsentiere das neue Frankfurt mithin den Reichtum des neuen Deutschland.

$\mathrm{Zu}$ den auffäligen Konstanten der innenpolitischen Diskussion in Frankreich nach 1871 gehören die Themen Schul-, Universitäts- und Bildungsreform ${ }^{52}$, nicht zuletzt deshalb, weil man in den entsprechenden deutschen Einrichtungen eine der Ursachen für die deutsche Überlegenheit zu sehen meinte. So bildet sich auch ein Typus der Bildungsinspektionsreise heraus, an der führende Gelehrte und Reformer wie Bréhier oder Lavisse beteiligt sind. In der großangelegten landeskundlichen Enquête Jules Hurets zu Beginn unseres Jahrhunderts steht dieser Aspekt ganz im Vordergrund. Der Autor und Journalist, sichtlich um Objektivităt und Ausgewogenheit des Urteils bemüht, geht von einer Korrelation zwischen dem allgemeinen Wohlstand, dem bürgerlichen Selbstbewußtsein und dem Zustand der Bildungs- und Sozialeinrichtungen aus. Frankfurt, inzwischen - wir befinden uns im Jahre 1901 - auf 290000 Einwohner angewachsen, mithin mehr als dreimal so groß wie 1860 , wächst weiter, " et elle prévoit qu'elle grandira davantage, et toujours plus " ${ }^{53}$. Was jedoch in den Augen des französischen Beobachters entscheidend ist, ist nicht das fast ungebremste Wachstum an sich, sondern die vorausschauende Planung und Organisation, die auch offentliche Bildungseinrichtungen und Stiftungen einbegreift : der Städel, das SenckenbergMuseum, die Académie des sciences sociales, wie er es nennt, Grundlage der späteren Universität, die Stadtbibliothek, aber auch sozialer Wohnungsbau, Waisenhäuser, Schulen, usw. Der Autor spricht von 100 privaten Vereinigungen, die sich soziale Aufgaben zum Ziel gesetzt haben, und einer koordinierenden Zentrale für private Fürsorge. Es ist sicherlich kein Zufall, daß innerhalb des umfangreichen Spektrums der besichtigten und beschriebenen deutschen Städte und Landschaften gerade Frankfurt den Ort einer exemplarischen Schulbesichtigung bildet : der Realschule, die als Musterschule unter der Leitung eines gewissen Professor Walter den Fremdsprachenunterricht durch die Einführung der direkten Methode zu reformieren versucht. Huret besucht die Französisch-Klassen und erweist sich als aufmerksamer und faszinierter Beobachter, der in dem praxis-

51. Ibid., p. 109.

52. Cf. op. cit. supra n. 27 und Christophe CharLe, " L'élite universitaire française et le système universitaire allemand (1880-1900)", in Transferts. Les relations inter-culturelles dans l'espace franco-allemand ( $x$ InIf-xIX' siècle), éds Michel Espagne, Michaell Werner, Paris, Recherche sur les civilisations A. D. P. F., 1988, p. 345-358.

53. J. HURET, op. cit. supra n. 11, p. 50. 
orientierten Denken ein Kennzeichen - und nicht das schlechteste des neuen Deutschland sieht. Die langen Ausführungen, deutlich dem französischen Publikum zur Nachahmung empfohlen, schließen mit einem Seitenhieb auf die ältere, ebenfalls deutsche Tradition der « philologues des langues romanes [...] incapables de prononcer deux phrases de langage courant $"{ }^{54}$. Das Schulthema rundet so den kosmopolitischen Anspruch $a b$, den der Beobachter in den vielfältigen Aktivităten und nicht zuletzt in der Uberlegenheit der Frankfurter Industrie und Handelshäuser zu erkennen glaubt. « Francfort, jadis ville libre », die " a gardé le sentiment de son indépendance et de sa force " $"$, erscheint als progressives Schaufenster des Deutschen Reiches, dessen negative Kehrseiten wie Dünkel und Militarismus hier praktisch ausgespart bleiben. Dies ist gewiß kein geringes Lob aus dem Munde eines der sachkundigsten Kulturkritiker seiner Zeit und seines Landes - am Vorabend jener Ereignisse, die eine hoffnungsvoll sich anbahnende Entwicklung für lange Zeit zunichte machen sollten. Und doch wirkt hier schon manches zeitgemäß.

Aber damit ist unser Thema auch vorerst abgeschlossen. Manche spezielle Fragestellung wäre nach diesem gewiß lückenhaften Überblick über ein spezielles, doch symptomatisches Kapitel deutsch-französischer Beziehungen noch interessant. So fällt z. B. auf, daß das Stichwort Paulskirche mit den damit verbundenen Ereignissen von 1848 so gut wie keine Rolle spielte. Der genannte Guide Joanne von 1863 macht zwischen 1830 und 1850 einen Sprung und bemerkt lakonisch - ohne die Paulskirche auch nur zu erwähnen - : « En 1850, Francfort vit rétablir l'ancienne Confédération germanique, qui avait été détruite en $1848 "{ }^{56}$. Auch die Messestadt Frankfurt steht nicht im Zentrum. Doch war dies nicht immer so. Und so wollen wir hier schließen wie weiland Rabelais in seinem Pantagruel, nachdem das Wichtigste geschafft ist :

"Or, Messieurs, vous avez ouy un commencement de l'Histoire horrifique [...] Ici je feray fin à ce premier livre ; la teste me faict un peu de mal, et je sens bien que les registres de mon cerveau sont quelques peu brouillez de ceste puree de septembre. Vous aurez le reste de l'histoire à ces foires de Francfort prochainement venantes... $"$ "

Friedrich WOLFZETTEL, Universität Frankfurt.

\footnotetext{
54. Ibid., p. 79.

55. Ibid., p. 50.

56. Guides Joanne, op. cit. supra n. 5, p. 50.

57. François Rabelais, Euvres complètes, éd. par Pierre Jourda, Paris, Garnier, 1962, t. I, p. 385.
} 